\title{
Simplified Measurement of Maximum Strength After Knee Surgery: Application-Based Knee-Training Device Compared to Isokinetic Testing
}

This article was published in the following Dove Press journal: Open Access Journal of Sports Medicine

\author{
Hauke Horstmann' \\ Pascal Medico ${ }^{2}$ \\ Florian Lasch iD $^{3}$ \\ Werner Krutsch ${ }^{4}$ \\ Thomas Sanjay Weber- \\ Spickschen $^{2}$ \\ 'Department of Orthopaedic Surgery, \\ Hannover Medical School, Hannover \\ 30625, Germany; ${ }^{2}$ Institute of Sports \\ Medicine, Hannover Medical School, \\ Hannover 30625, Germany; ${ }^{3}$ Institute for \\ Biometry, Hannover Medical School, \\ Hannover 30625, Germany; ${ }^{4}$ Trauma \\ Department, University of Regensburg, \\ Regensburg 93053, Germany
}

Context: Isokinetic testing is used as a standard tool in measuring strength in professional athletes. It is often used to evaluate improvement during rehabilitation. The disadvantages of isokinetic testing include its costs, the fact that it is not portable, and its risk of injury, which makes it not suitable for early postoperative rehabilitation.

Hypothesis: The aim of this study was to investigate the relationship between the results of the isokinetic testing and the measurements of an application-based knee-training device.

Study Design: Exploratory diagnostic study.

Methods: In this monocentric study, 100 subjects performed an isokinetic maximum strength examination and an assessment on the application-based knee-training device in a randomized order. The isokinetic testing was based on the Swiss Olympic protocol with 3 sets of 5 repetitions of maximum strength testing for flexion and extension. The subjects consisted of 50 healthy professional athletes and 50 healthy recreational athletes, half male and half female, between the ages of 18 to 30 years old.

Results: No medical or technical issues were reported. The analysis of the relationship between application-based knee-training device and extension showed a Pearson correlation coefficient of $r=0.667$ for the left knee and $r=0.604$ for the right knee. For flexion, the Pearson correlation coefficient was $r=0.640$ for the left side and $r=0.673$ for the right side. When strength measured by the application-based knee-training device was adjusted for height and weight of the subjects, the Pearson correlation was even stronger (extension left: $\mathrm{r}=0.727$, right: $\mathrm{r}=0.689$; flexion left: $\mathrm{r}=0.641$, right: $\mathrm{r}=0.711$ ).

Conclusion: The study shows a moderate to high correlation between isokinetic testing and the application-based knee-training device. These results suggest that the application-based knee-training device is effective for early strength rehabilitation without the risk of injury.

Keywords: knee, isokinetic, app-based rehabilitation, exergame

\section{Introduction}

With the increasing amount of knee-related surgery including total knee arthroplasties, rehabilitation costs amount to a multimillion dollar market. ${ }^{1,2}$ The time required to regain normal gait and balance is reduced by an early initiation of rehabilitation. ${ }^{3}$ Studies have shown good clinical results and high patient satisfaction with a shortened length of stay in hospital. ${ }^{4-6}$ Therefore, an accelerated perioperative treatment including an early rehabilitation program has a positive impact for both the health system and the patient. $^{7}$

Considering both cost-effectiveness and the interest in enhanced recovery pathways for the further development of outpatient surgery protocols, ${ }^{8}$ there is an increasing need for self-explanatory rehabilitation devices. The development of self-explanatory
Correspondence: Hauke Horstmann Diakovere - Annastift, Department of Orthopaedic Surgery, Hannover Medical School, Anna-von-Borries-Street I-7, Hannover 30625, Germany

Tel +49 5115354568

Email hauke.horstmann@diakovere.de 
rehabilitation devices requires the use of modern technology. Multiple studies have suggested that the use of video games in rehabilitation of various health disorders may be a promising approach. ${ }^{9,10}$ Different groups have used computer game-associated orthopaedic rehabilitation-also called exergames - to enhance independent rehabilitation. For example, the Nintendo Wii Fit has been successfully introduced to the post-operative program of anterior cruciate ligament reconstruction. ${ }^{9}$ Based on the Nintendo Wii Remote and Wii Fit balance board controllers, Costa et al have developed a computer game specifically designed for orthopaedic rehabilitation. ${ }^{11}$ Two knee-related rehabilitation devices were developed and validated, namely, the Locomo Scan (ALCARE Co., Ltd., Tokyo, Japan) ${ }^{12}$ and the application-based knee-training device (KT). ${ }^{13,14}$

The development and fulfilment of a personalized rehabilitation protocol requires defined milestones. ${ }^{15}$ Progress is tracked by measuring strength of the muscles around the knee. Extension and flexion are particularly important when recovering from sports-related injuries as well as from surgery like arthroplasty at the knee joint. Assessments of strength are mostly done by isokinetic testing as the current gold standard. Unfortunately, isokinetic testing has the disadvantages of being difficult to perform, expensive, and not portable. Clinical tests like "timed up and go" are also commonly used but they are often too complex to assess extension or flexion. A simplified and objective measurement of strength - especially in the early postoperative phase - would be a valuable clinical tool.

Application-based knee-training devices have the advantage of being cost-effective, portable, and safe. ${ }^{13,14}$ The knee-training device introduces the joy of an exergame to orthopaedic rehabilitation and provides a protocol of the training progress. In summary, the knee-training device has great potential and has the advantage of topics in the abstracts above. However, strength training on the knee-training device needs to be compared to strength examination on an isokinetic dynamometer-the gold standard of strength assessment. ${ }^{16,17}$ This is an exploratory study evaluating the correlation of KT measurements and isokinetic testing not testing a formal hypothesis confirmatory. The underlying (informal) hypothesis was that the KT measurements and the isokinetic testing show a good linear correlation.

\section{Methods}

\section{Experimental Subjects}

In a monocentric study, 100 subjects underwent an isokinetic maximum strength examination and an assessment on the KT in randomized order. The isokinetic testing was based on the Swiss Olympic protocol with 3 sets of 5 repetitions of maximum strength testing for flexion and extension. The subjects consisted of 50 healthy professional athletes and 50 healthy recreational athletes between the ages of 18 to 30 years old. The sample size and division in professional/recreational athletes were feasibility driven. The sample size and division in professional/recreational athletes were feasibility driven. Professional and recreational athletes were included to display a range of strength. Each group was $50 \%$ male. Ethical approval was granted by the Ethics Committee of Hannover Medical School, Germany (Nr. 3103-2016). The tests were performed at the Olympic training centre in Hannover. The subjects were asked to take part in the study in the daily routine. Eligible subjects were capable to exercise. Exclusion criteria were a missing capability to exercise and kneerelated injuries in particular. Medical aid was provided to meet human subject protection. In addition, all subjects were informed of their right to end participation in the study at any time point. All subjects were informed of their rights and written consent was obtained before beginning the tests.

\section{Knee-Training Device and Application}

The KT including application has been previously described by Weber-Spickschen et al ${ }^{13,14}$ In brief, the body of the KT is 18 (length) x 12 (width) x $8 \mathrm{~cm}$ (height) and is supplied with four AA batteries. It is composed of three piezoresistive force sensors (FlexiForce A201, Tekscan, South Boston, USA), two LEDs, and an Arduino electric circuit board (Arduino, USA). The body of the KT consists of polyoxymethylene copolymer (POM-C). The KT is placed under the popliteal fossa for training sessions.

The application consists of two parts. The first part of the application contains a high striker exercise. Subjects are instructed to extend their knee and therefore press their popliteal fossa as hard as possible against the knee-training device for $5 \mathrm{~s}$ to achieve a maximum score. The maximum force of the knee extension is measured and recorded.

The second part of the application is a game, which is intended to improve the users' coordination for achieving better rehabilitation results. In the application, the user is piloting a plane for $100 \mathrm{~s}$. Extending the knee and pressing the popliteal fossa in a dosed and dynamic manner against the knee device achieves steering of the plane. In the game, the plane flies through an animated sky with balloons. The force of the popliteal fossa on the device guides the height of the aircraft in the sky. The stronger the force, the higher the aeroplane will fly. In order to achieve a higher score, the 
plane needs to exactly follow a designated path of balloons. By following the path of balloons, balloons burst with the front propeller. The player of the game gets points with every burst balloon.

The training time, frequency of training, the maximum knee extension force, the accuracy of quadriceps muscle activation, and the final scores are recorded in the application. ${ }^{13,14}$ In this study, the higher of the two maximum force measurements were compared to the isokinetic testing.

\section{Isokinetic Testing}

A number of different tests have been used to determine maximum strength using isokinetic testing. The most common method measuring extension and flexion strength of the lower limb was used. ${ }^{18,19}$ Testing was performed on a CON-TREX Multi-Joint System (CMV AG, Dübendorf Switzerland) ${ }^{18}$ and included measurement of the point of rotation and lever arm. Knee strength was assessed by using isokinetic parameters at angular velocities of $60 \%$ (5 repetitions) with 3 sets and a one-minute break in between sets. The peak torque value was determined. The results were adjusted with the lever arm of the force and the body weight of the patients. The outcome is presented in Newton per kilogram body weight and compared to the strength of the healthy limb.

\section{Data Analysis}

The association of isokinetic testing and the KT regarding tension and flexion was analysed separately using mixed models for repeated measurements: the isokinetic testing result was modelled as dependent variable and the KT result was modelled as independent variable. The tested knee was modelled as a repeated factor within each subject using a compound symmetry covariance matrix to adjust for correlated measurements of the right and left knee of each patient. In additional analyses, the independent factors height and weight were included separately and combined as fixed factors into the analysis for adjustment. Based on the Akaike information criterion (AIC), the respective models with the best fit were chosen as a prediction model for extension and flexion. A modified Bland-Altman plot was used to illustrate the errors of the prediction model from the true isokinetic testing results. ${ }^{20}$

Baseline characteristics of the study participants have been analysed using appropriate descriptive statistics (mean and standard deviation for continuous endpoints, absolute and relative numbers for categorical endpoints).
No (statistical) hypothesis testing has been performed, since the study was not designed to analyse any hypotheses regarding the baseline characteristic of the population. Professional and recreational athletes have been included to ensure sufficient heterogeneity in the study population and increase external validity, there was no allocation of "professional" or "recreational" but a stratified inclusion of athletes who had the respective characteristic.

Additionally, the Pearson correlation was used to describe the linear relationship between isokinetic testing and both KT testing and the prediction model based on the KT and covariates. ${ }^{21}$ Since measurements of the left and right knee within each patient are correlated, Pearson correlation was calculated separately using either only measurements of the left or the right knees. Continuous variables are reported as mean \pm standard deviation and categorical variables are reported by absolute and relative frequencies.

A two-sided significance level of 5\% was used for the evaluation of statistical significance. Since no prespecified primary hypothesis was tested, all p-values and statistical significances are considered to be exploratory and not confirmatory.

\section{Results}

This study sought to compare the measurements of the KT with the results of the isokinetic testing when used by healthy subjects. Characteristics of the study population are shown in Table 1. No medical or technical issues were reported during the course of the study and there are no missing values for any of the analysed variables. The basic strength measurements are shown in Table 2.

Based on the correlation analysis, the KT presented a correlation coefficient of $\mathrm{r}=0.667$ for the left side and $\mathrm{r}=0.604$ for the right side in extension (see Figure 1). For flexion, the correlation coefficient for the left side was $\mathrm{r}=0.640$ and for the right side was $\mathrm{r}=0.673$ (see Figure 2). Subanalysis did not show any significant difference between professional and recreational athletes.

Both for extension and flexion, the model including both height and weight as additional fixed effects showed the best AIC and were chosen for prediction (see Table 3 for the fixed effects estimates of the respective models). For extension, the model-derived formula to predict isokinetic testing by KT testing $(\mathrm{kg})$, weight $(\mathrm{kg})$, and height (m) is given by:

Isokinetic (predicted) $=-39.96+5.85 \mathrm{KT}+83.30$ height +4.54 weight 
Table I Baseline Characteristics of the Study Population

\begin{tabular}{|l|l|l|l|}
\hline Characteristic & Professional Athletes & Recreational Athletes & Total Athletes \\
\hline No. of patients & 50 & 50 & 100 \\
Age (year) & $21.8( \pm 2.9)$ & $23.2( \pm 3.4)$ & $22.49( \pm 3.26)$ \\
Sex (no. of females) & $25(50 \%)$ & $25(50 \%)$ & $50(50 \%)$ \\
Weight (kg) & $75.1( \pm 18.3)$ & $73.8( \pm 11.8)$ & $74.44( \pm 15.39)$ \\
Height (cm) & $177.2( \pm 9.8)$ & $176.3( \pm 8.2)$ & $176.75( \pm 9.06)$ \\
Body-mass index & $23.6( \pm 3.7)$ & $23.7( \pm 3.2)$ & $23.65( \pm 3.46)$ \\
Hours of training per week & $14.4( \pm 5.0)$ & $6.1( \pm 4.0)$ & $10.24( \pm 6.17)$ \\
\hline
\end{tabular}

Note: Descriptive statistics are reported as mean ( \pm standard deviation) for continuous variables and absolute frequency (relative frequency) for categorical variables.

Table 2 Results of Strength Measurements

\begin{tabular}{|l|l|l|l|}
\hline Characteristic & Right Knee & Left Knee & Total \\
\hline Extension (N) & $578.23( \pm 149.51)$ & $570.25( \pm 146.97)$ & $574.24( \pm 147.93)$ \\
Flexion (N) & $336.63( \pm 104.33)$ & $321.69( \pm 100.76)$ & $329.16( \pm 102.58)$ \\
KT (kg) & $21.91( \pm 5.91)$ & $22.16( \pm 5.83)$ & $22.04( \pm 5.86)$ \\
\hline
\end{tabular}

Note: All variables are reported with mean ( \pm standard deviation).

For flexion, isokinetic testing is predicted by the KT testing $(\mathrm{kg})$, weight $(\mathrm{kg})$ and height $(\mathrm{m})$ by:

Isokinetic (predicted) $=-247.77+6.38 \mathrm{KT}+195.15$ height +1.23 weight

When the KT results were transformed using the prediction formulas, including an adjustment for height and weight of the subjects, the Pearson correlation of isokinetic and KT was even stronger than unadjusted (extension left: $\mathrm{r}=0.727$, right: $r=0.689$; flexion left: $r=0.641$, right: $r=0.711$ ). Figures 3 and 4 illustrate that the prediction formulas work equally well for right and left knee. However, analysing the deviation of the predicted isokinetic value from the true value, as illustrated in Figures 5 and 6 , indicates systematic bias in the prediction,

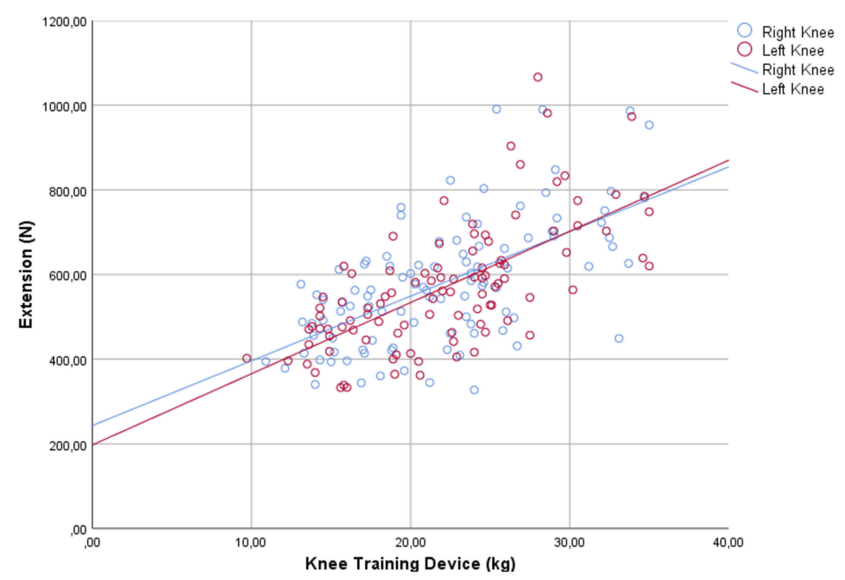

Figure I Linear relationship of isokinetic results for extension and knee-training device results separately for right and left knee. Pearson correlation for the left and right knee is 0.667 and 0.604 . which is present despite adjustment for height and weight. While on average the bias is zero, large values of isokinetic testing tend to be underestimated while small values tend to be overestimated.

\section{Discussion}

The major findings of the present study are the moderate to high Pearson correlation of KT and isokinetic extension and flexion measurements. These positive results are corroborated by mean differences between predicted and true isokinetic measurements ranging from $+8.26 \mathrm{~N}$ to $-8.26 \mathrm{~N}$ (with limits of agreement ranging from $+207.75 \mathrm{~N}$ to $-217.19 \mathrm{~N})$. This accuracy is precise enough to be effective

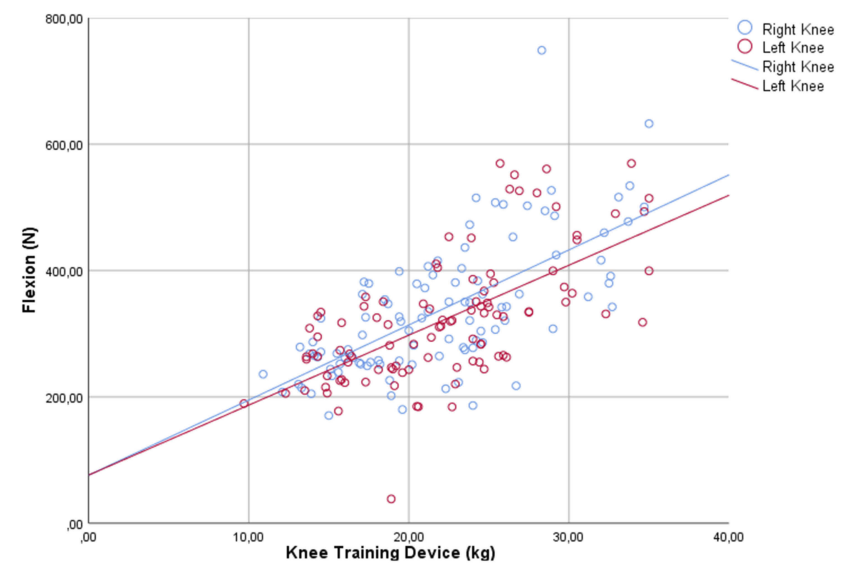

Figure 2 Linear relationship of isokinetic results for flexion and knee-training device results separately for right and left knee. Pearson correlation for the left and right knee is 0.640 and 0.673 . 
Table 3 Parameter Estimates of the Statistical Model

\begin{tabular}{|l|l|l|l|l|l|}
\hline & Parameter & Estimate & Standard Error & p-value & 95\% Confidence Interval \\
\hline \multirow{2}{*}{ Extension } & Constant & -39.96 & 243.23 & 0.870 & {$[-522.73,442.80]$} \\
& Weight $(\mathrm{kg})$ & 4.54 & 1.00 & 0.000 & {$[2.56,6.53]$} \\
& Height $(\mathrm{m})$ & 83.30 & 165.74 & 0.616 & {$[-245.62,412.22]$} \\
& Knee training device result $(\mathrm{kg})$ & 5.85 & 1.97 & 0.003 & {$[1.95,9.74]$} \\
\hline \multirow{2}{*}{ Flexion } & Constant & -247.77 & 173.44 & 0.156 & {$[-592.06,96.52]$} \\
& Weight $(\mathrm{kg})$ & 1.23 & 0.72 & 0.089 & {$[-0.19,2.65]$} \\
& Height $(\mathrm{m})$ & 195.15 & 118.25 & 0.102 & {$[-39.55,429.85]$} \\
& Knee training device result $(\mathrm{kg})$ & 6.38 & 1.46 & 0.000 & {$[3.5,9.26]$} \\
\hline
\end{tabular}

in postoperative rehabilitation, especially considering the direction of impact of the KT to show improvement in muscle activation and strength in a motivating manner. Altogether, our results indicate that longitudinal measurements (i.e. of early strength rehabilitation) can be performed with the KT.

The results from our study are comparable to Omori et al who used a similar device but without an applicationbased game. ${ }^{22}$ Omori et al tested their knee-training device QTM on 24 subjects and obtained a correlation between the device and the isokinetic measurements of $r=0.69$ (and 0.82 in the second measurement).

Muscle activation shortly after knee operations has been shown to be important for recovery. ${ }^{23}$ Simultaneously, trends of fast-tracking rehabilitation after surgery require patients to move more quickly into an outpatient status. ${ }^{8}$ With the application-based KT patients can begin their rehabilitation early and at home. Previous studies did not show a superior rehabilitation in an inpatient setting com-

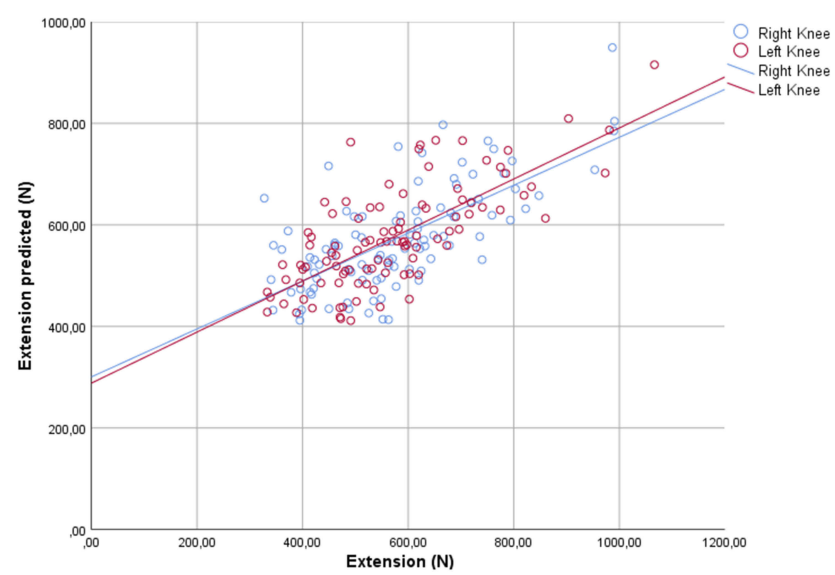

Figure 3 Linear relationship of isokinetic results for extension and predicted results based on KT results, weight and height separately for right and left knee. Pearson correlation coefficients for the left and right knee are 0.727 and 0.689 . pared to a home-based or outpatients controlled setting. ${ }^{24,25}$ Exergames such as the application-based KT have the potential to have a large impact on home-based rehabilitation. Previous exergame studies for orthopedic rehabilitation also showed promising results ${ }^{9,11}$ mostly combining a motivating aspect with muscle activation. However, as opposed to these former exergames, our KT allowed actual resistance exercises to be performed.

Based on the technique of the KT as well as from isokinetic testing, inaccuracies in measurement were identified. Despite the fact that the tests were done with the highest possible precision, there was a potential chance of mal-positioning of the application-based KT under the popliteal fossa. Similar, the mal-positioning of the subjects while performing the isokinetic testing ${ }^{26}$ can lead to inaccurate results.

The clinical significance of the comparison of KT and isokinetic testing is the potential of implementing this exergaming device to the clinical routine. ${ }^{22}$

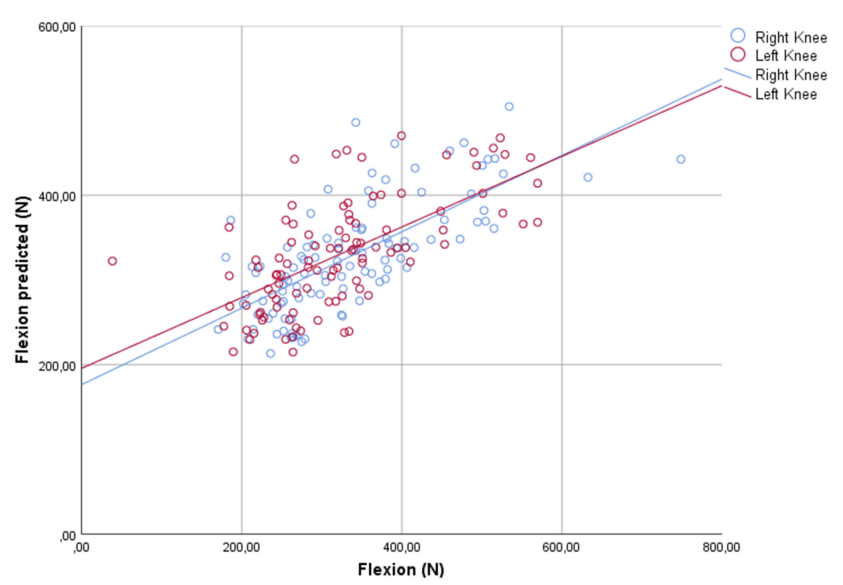

Figure 4 Linear relationship of isokinetic results for flexion and predicted results based on KT results, weight, and height separately for right and left knee. Pearson correlation coefficients for the left and right knee are $0.64 \mathrm{I}$ and $0.7 \mathrm{I}$. 


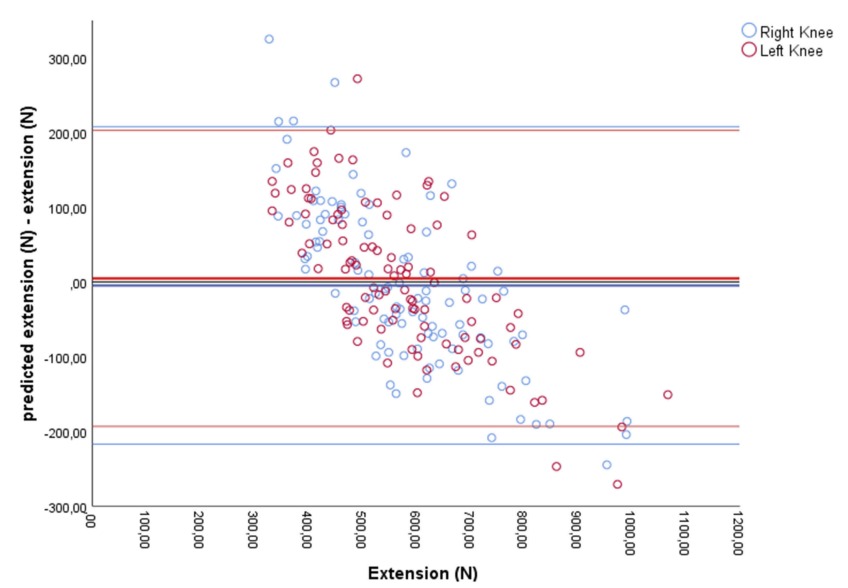

Figure 5 Modified Bland-Altman plot of the difference of the predicted isokinetic extension value from the true isokinetic extension value. Mean differences and $95 \%$ limits of agreement calculated by mean difference \pm 1.96 standard deviation are given separately for left $(-193.3 ; 202.73)$ and right knee $(-217.19 ; 207.75)$. Mean difference is $4.72( \pm 101.03)$ for the left knee and $-4.72( \pm 108.40)$ for the right knee.

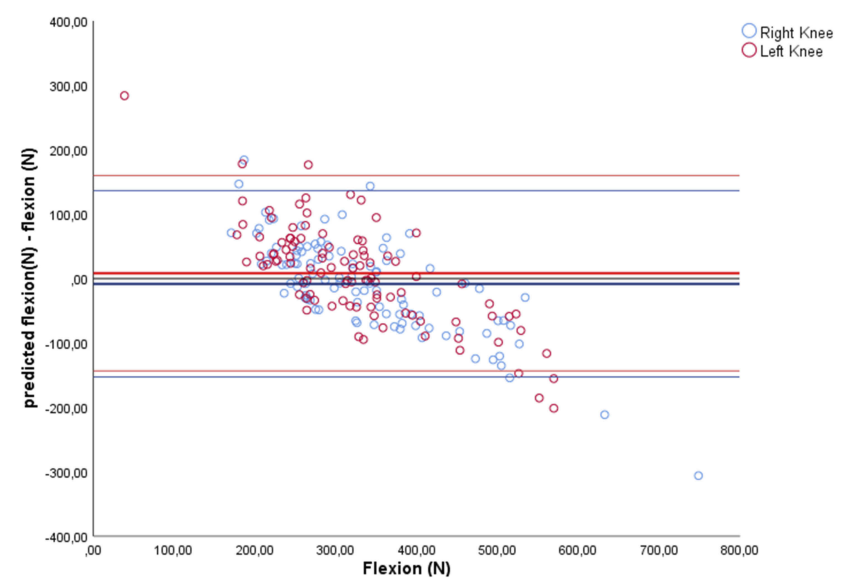

Figure 6 Modified Bland-Altman plot of the deviation of the predicted isokinetic flexion value from the true isokinetic flexion value modified. Mean differences and $95 \%$ limits of agreement calculated by mean difference \pm 1.96 standard deviation are given separately for left $(-143.40 ; 159.93)$ and right knee $(-152.83 ; 136.31)$. Mean difference is $8.26( \pm 77.38)$ for the left knee and $-8.26( \pm 73.76)$ for the right knee.

Future studies should validate the data-derived prediction model in an independent sample and focus on the introduction of the KT into clinical protocols. For the sake of addressing certain muscle groups, it is important to define particular seating positions for special exercises (i.e. for the quadriceps- or hamstrings muscle group).

\section{Conclusion}

This study confirms a moderate to high correlation between isokinetic testing and the KT. The results indicate that followup with the low-cost KT improves early strength rehabilitation without the risk of injury. The measurement of maximum strength after knee operation can be simplified.

\section{Disclosure}

Dr. Horstmann has no shares in the Genusport GmbH or any other company/organization which is involved in the project. Dr. Horstmann reports grants from Röver Stiftung, during the conduct of the study. Dr Weber-Spickschen reports grants from Röver Stiftung, during the conduct of the study; has shares in GenuSport $\mathrm{GmbH}$; and has a patent for GenuSport pending. The authors report no other conflicts of interest in this work.

\section{References}

1. Kurtz S, Ong K, Lau E, Mowat F, Halpern M. Projections of primary and revision hip and knee arthroplasty in the United States from 2005 to 2030. J Bone Joint Surg Am. 2007;89(4):780-785.

2. Kurtz SM, Lau E, Ong K, Zhao K, Kelly M, Bozic KJ. Future young patient demand for primary and revision joint replacement: national projections from 2010 to 2030. Clin Orthop Relat Res. 2009;467 (10):2606-2612.

3. Labraca NS, Castro-Sanchez AM, Mataran-Penarrocha GA, Arroyo-Morales M, Sanchez-Joya Mdel M, Moreno-Lorenzo C. Benefits of starting rehabilitation within 24 hours of primary total knee arthroplasty: randomized clinical trial. Clin Rehabil. 2011;25(6):557-566.

4. Andreasen SE, Holm HB, Jorgensen M, Gromov K, KjaersgaardAndersen P, Husted H. Time-driven Activity-based Cost of FastTrack Total Hip and Knee Arthroplasty. J Arthroplasty. 2017;32 (6):1747-1755.

5. Husted H. Fast-track hip and knee arthroplasty: clinical and organizational aspects. Acta Orthop Suppl. 2012;83(346):1-39.

6. Husted H, Hansen HC, Holm G, et al. Accelerated versus conventional hospital stay in total hip and knee arthroplasty III: patient satisfaction. Ugeskr Laeger. 2006;168(22):2148-2151.

7. Larsen K, Sorensen OG, Hansen TB, Thomsen PB, Soballe K. Accelerated perioperative care and rehabilitation intervention for hip and knee replacement is effective: a randomized clinical trial involving 87 patients with 3 months of follow-up. Acta Orthop. 2008;79(2):149-159.

8. Schotanus MGM, Bemelmans YFL, Grimm B, Heyligers IC, Kort NP. Physical activity after outpatient surgery and enhanced recovery for total knee arthroplasty. Knee Surg Sports Traumatol Arthrosc. 2017;25(11):3366-3371.

9. Baltaci G, Harput G, Haksever B, Ulusoy B, Ozer H. Comparison between Nintendo Wii Fit and conventional rehabilitation on functional performance outcomes after hamstring anterior cruciate ligament reconstruction: prospective, randomized, controlled, doubleblind clinical trial. Knee Surg Sports Traumatol Arthrosc. 2013;21 (4):880-887.

10. Pietrzak E, Cotea C, Pullman S. Using commercial video games for upper limb stroke rehabilitation: is this the way of the future? Top Stroke Rehabil. 2014;21(2):152-162.

11. Costa C, Tacconi D, Tomasi R, Calva F, Terreri V. RIABLO: A game system for supporting orthopedic rehabilitation. 2013.

12. Narumi K, Funaki Y, Yoshimura N, et al. Quadriceps muscle strength reference value as index for functional deterioration of locomotive organs: Data from 3617 men and women in Japan. J Orthop Sci. 2017;22(4):765-770.

13. Weber-Spickschen TS, Colcuc C, Hanke A, Clausen JD, James PA, Horstmann H. Fun During Knee Rehabilitation: Feasibility and Acceptability Testing of a New Android-Based Training Device. Open Med Inform J. 2017;11:29-36. 
14. Horstmann H, Krost E, Welke B, et al. The determination of the validity of an application-based knee-training device. Assist Technol. 2018.

15. Gokeler A, Dingenen B, Mouton C, Seil R. Clinical course and recommendations for patients after anterior cruciate ligament injury and subsequent reconstruction: A narrative review. EFORT Open Rev. 2017;2(10):410-420.

16. Danneskiold-Samsoe B, Bartels EM, Bulow PM, et al. Isokinetic and isometric muscle strength in a healthy population with special reference to age and gender. Acta Physiol (Oxf). 2009;197 Suppl 673:1-68.

17. Gaines JM, Talbot LA. Isokinetic strength testing in research and practice. Biol Res Nurs. 1999;1(1):57-64.

18. Kim do K, Park WH. Sex differences in knee strength deficit 1 year after anterior cruciate ligament reconstruction. J Phys Ther Sci. 2015;27(12):3847-3849.

19. Kannus P. Normality, variability and predictability of work, power and torque acceleration energy with respect to peak torque in isokinetic muscle testing. Int J Sports Med. 1992;13(3):249-256.

20. Bland JM, Altman DG. Statistical methods for assessing agreement between two methods of clinical measurement. Lancet. 1986;1 (8476):307-310.
21. Cohen J. A power primer. Psychol Bull 1992 Jul;112(1):155-159.

22. Omori G, Koga Y, Tanaka M, et al. Quadriceps muscle strength and its relationship to radiographic knee osteoarthritis in Japanese elderly. J Orthop Sci. 2013;18(4):536-542.

23. Mizner RL, Petterson SC, Stevens JE, Vandenborne K, SnyderMackler L. Early quadriceps strength loss after total knee arthroplasty. The contributions of muscle atrophy and failure of voluntary muscle activation. J Bone Joint Surg Am. 2005;87(5):1047-1053.

24. Han AS, Nairn L, Harmer AR, et al. Early rehabilitation after total knee replacement surgery: a multicenter, noninferiority, randomized clinical trial comparing a home exercise program with usual outpatient care. Arthritis Care Res (Hoboken). 2015;67(2):196-202.

25. Papalia R, Vasta S, Tecame A, D’Adamio S, Maffulli N, Denaro V. Home-based vs supervised rehabilitation programs following knee surgery: a systematic review. Br Med Bull. 2013;108:55-72.

26. Mayer F, Horstmann T, Bäurle W, Grau S, Handel M, Dickhuth HH. Diagnostics with isokinetic devices in shoulder measurements potentials and limits. Isokinetics and Exercise Science. 2001;9 (1):19-25.

\section{Publish your work in this journal}

Open Access Journal of Sports Medicine is an international, peerreviewed, open access journal publishing original research, reports, reviews and commentaries on all areas of sports medicine. The manuscript management system is completely online and includes a very quick and fair peer-review system. Visit http://www.dovepress. com/testimonials.php to read real quotes from published authors. 